\title{
Miomas sintomáticos: ¿miomectomía o embolización de las arterias uterinas?
}

\author{
Vanessa García D. ${ }^{1}$, Sofía Elisa Cernadas $P{ }^{1}{ }^{1}$,Vanesa Buján $C{ }^{1}$, Roberto González \\ B. ${ }^{1}$, Paula González G. ${ }^{1}$, Elena Cerviño G. ${ }^{1}$, Emilio Couceiro N. 1', Carlos Nicolás Ló- \\ pez R. ${ }^{1}$, Sonia Cea P. ${ }^{2}$, Carmen Neches R. 2
}

\footnotetext{
${ }^{1}$ Servicio de Ginecología y Obstetricia, ${ }^{2}$ Servicio de Radiología Intervencionista, Complejo Hospitalario Universitario de Vigo. Pontevedra, España.
}

\section{RESUMEN}

Objetivo: Evaluar las complicaciones asociadas a la miomectomía laparotómica y a la embolización de las arterias uterinas en mujeres con miomas sintomáticos. Métodos: Estudio descriptivo restrospectivo efectuado en el Complejo Hospitalario Universitario de Vigo desde el año 2008 al 2010. La población en estudio la conformaron aquellas mujeres sometidas a una miomectomía laparotómica o a una embolización de las arterias uterinas. Se recopilaron las complicaciones registradas en las historias clínicas de las pacientes tras las técnicas. Resultados: La tasa de complicaciones en el grupo de las pacientes sometidas a miomectomía fue de 15,2\%; las más frecuentes fueron fiebre, anemia, necesidad de trasfusión y hematoma subcutáneo. La tasa de complicaciones en el grupo de embolización fue de 4,5\% recogiéndose casos de síndrome postembolización, mioma parido y un caso de histerectomía. Conclusión: La adecuada indicación de cada técnica es fundamental para tener una baja tasa de complicaciones. Se debe tener en cuenta la sintomatología, tipo y número de miomas, edad y deseo genésico de cada paciente.

\section{PALABRAS CLAVE: Embolización de arteria uterina, miomectomía, mioma uterino}

\section{SUMMARY}

Objective: This study was undertaken to evaluate the complications after laparotomy myomectomy and uterine artery embolization in women with symptomatic uterine leiomyoma. Methods: This was a descriptive and retrospective study of patients who were treated with embolization and myomectomy for leiomyomas from 2008-2010. Complications were measured. Results: The rate of complications collected in the myomectomy group was $15.2 \%$; there were cases of fever, anemia, need for transfusion and subcutaneous hematoma. The rate of complications collected in the embolization group was $4.5 \%$; there were cases of embolization syndrome, transcervical fibroid expulsion and a case of hysterectomy. Conclusion: The suitable indication of every tecnic is fundamental to have a low rate of complications. It is necessary to bear in mind the symptomatology, type and number of myomas and age and reproductive desire of each patient.

KEY WORDS: Uterine artery embolization, myomectomy, uterine leiomyoma 


\section{INTRODUCCIÓN}

El mioma uterino es el tumor benigno ginecológico más frecuente, presente hasta en el $40 \%$ de las mujeres. En el $30 \%$ de los casos los miomas se asocian a alteraciones menstruales, disconfort pélvico, dolor e infertilidad (1). Su prevalencia en la gestación es del $10 \%$ y son causa del $2-3 \%$ de los casos de infertilidad. El manejo activo de los miomas maximiza la tasa de reproducción de forma significativa (2). La localización y el tamaño son aspectos condicionantes. Las opciones terapéuticas incluyen tratamientos médicos, miomectomía, la embolización de las arterias uterinas (EAU) y la histerectomía.

La EAU está indicada en mujeres con miomas sintomáticos y sin deseo gestacional, siendo su uso controvertido en pacientes con deseo reproductivo. Las contraindicaciones de la EAU son: el embarazo, la infección génito-urinaria activa, la sospecha de enfermedad neoplásica, los miomas calcificados o necrosados, y los derivados de la técnica, coagulopatías, insuficiencia renal y alergia al contraste iodado. Como complicaciones se han descrito el riesgo de infección, sepsis, histerectomía y fallo ovárico precoz (1).

La miomectomía se considera el tratamiento estándar en pacientes con deseo genésico, aunque se asocia a recurrencias, sangrado, adherencias y rotura uterina en gestaciones futuras (3).

El objetivo de este estudio es evaluar las complicaciones asociadas a ambas técnicas en mujeres con miomas sintomáticos.

\section{PACIENTES Y MÉTODO}

Estudio descriptivo retrospectivo, efectuado en el Complejo Hospitalario Universitario de Vigo, durante el periodo 2008-2010. La población en estudio fueron pacientes sometidas a miomectomía laparotómica ó a la embolización de las arterias uterinas. Las variables analizadas fueron: edad, paridad, indicación de intervención, y número, tipo y tamaño de los miomas, también las complicaciones posteriores a la intervención, tiempo de hospitalización y respuesta al tratamiento. Los datos se procesaron mediante el programa estadístico SPSS versión 18. El valor $p<0,05$ indicó diferencia estadísticamente significativa.

\section{RESULTADOS}

El número total de pacientes incluidas en el estudio fue de 155 casos: 67 miomectomías y 88
EAU. La edad media de las pacientes fue de 35 años (rango: 23-46 años) en el grupo de la miomectomía y de 45 años (rango: 25-56 años) en el grupo de la embolización. En relación a las gestaciones previas, en el primer grupo el porcentaje mayor correspondió a pacientes nuligestas (68\%) y en el segundo grupo a multigestas (70\%). El 65,2\% de las pacientes en las que se realizó miomectomía tenían un mioma único, el $71,9 \%$ de las pacientes con EAU presentaban miomas múltiples. En ambos grupos el tipo de mioma más frecuente fue el intramural representado un 56,1\% en el grupo de la miomectomía y $37,9 \%$ en el de la EAU. En el tamaño medio de los miomas no hubo diferencias significativas entre ambos grupos, siendo $8,46 \mathrm{~cm}$ en el grupo miomectomía y $7,3 \mathrm{~cm}$ en el grupo EAU. En una de cada cinco miomectomías (19,7\%) se produjo la entrada en la cavidad uterina .

La estancia media hospitalaria en el grupo de las pacientes sometidas a miomectomía fue casi el triple que en el grupo de la EAU (Tabla I). La estancia media hospitalaria fue de 6,3 días en el grupo de miomectomía y 2,8 en el de la EAU.

La principal indicación en el grupo miomectomía fue la sintomatología compresiva (40\%) y en segundo lugar (28\%) el sangrado uterino anormal. En el grupo de pacientes sometidas a embolización, en el $59 \%$ de los casos la indicación de la técnica fue por sangrado anormal seguida por la sintomatología compresiva (28\%). No se hallaron diferencias estadísticamente significativas entre estas variables.

La tasa global de complicaciones en el grupo de la miomectomía fue del 15,2\%; las más frecuentes fueron fiebre, anemia, necesidad de trasfusión y el hematoma subcutáneo. En el grupo de la embolización fue del $4,5 \%$ registrándose un caso de síndrome postembolización, otro de mioma parido y destacando un caso de histerectomía.

La evolución fue favorable en el $84 \%$ de las pacientes miomectomizadas y en el $92,3 \%$ de los casos de embolización. El dolor abdominal persistió en el $3,2 \%$ y $1 \%$ de las pacientes respectivamente. En lo referente al sangrado uterino anormal, se mantuvo en el $3,2 \%$ y $2,6 \%$ de las pacientes. En el grupo de la EAU, la reembolización fue necesaria en el $1,3 \%$ de las pacientes y la histerectomía posterior en el 3,8\% $(n=3)$ debido a la persistencia de la sintomatología (dos de los casos por no cese de la metrorragia y en un caso por endometritis complicada con cuadro séptico) (Tabla II).

Han sido registrados cinco casos de gestaciones tras la miomectomía (sólo tres llegaron a término y dos abortos). No hay ninguna gestación en el grupo de las EAU (Tabla II). 
Tabla I

CARACTERÍSTICAS PREVIAS A LA INTERVENCIÓN EN CADA UNO DE LOS GRUPOS Y TIEMPO DE HOSPITALIZACIÓN

\begin{tabular}{lll}
\hline & Grupo & $\begin{array}{l}\text { Grupo } \\
\text { Embolización }\end{array}$ \\
\hline Edad media & Miomectomía & 45 años (25-56). \\
Gestación previa & 35 años (23-46) & $30 \%$ nuligesta \\
Número de miomas & $68 \%$ nuligesta & $70 \%$ multigesta \\
& $32 \%$ multigesta & Únicos : $28,1 \%$ \\
Tipo de miomas & Único: $65,2 \%$. & Múltiples: $71,9 \%$. \\
& Múltiples: 33,3\%. & $37,9 \%$ intramural \\
& $56,1 \%$ intramural & $2,3 \%$ submucoso \\
Tamaño medio del mioma & $1,5 \%$ submucoso & $11,5 \%$ subseroso \\
Entrada a cavidad uterina & $31,8 \%$ subseroso & $20,7 \%$ I+SS \\
Días de hospitalización & $10,6 \%$ I+SS & $7,3 \mathrm{~cm}$ \\
\hline
\end{tabular}

I: mioma intramural. SS: mioma subseroso.

Tabla II

EVOLUCIÓN, COMPLICACIONES TRAS LA INTERVENCIÓN, NECESIDAD DE REINTERVENCIÓN Y TASA DE GESTACIÓN POSTERIOR EN CADA GRUPO

\begin{tabular}{lcc}
\hline & Miomectomía & Embolización \\
\hline Evolución favorable & $84,1 \%$ & $92,3 \%$ \\
Dolor abdominal & $3,2 \%$ & $1 \%$ \\
Sangrado anormal & $3,2 \%$ & $2,6 \%$ \\
Reembolización & 0 & $1,3 \%$ \\
Histerectomía posterior & 0 & $3,8 \%(3)$ \\
Gestación posterior & 5 (2 abortos) & 0 \\
\hline
\end{tabular}

\section{DISCUSIÓN}

La miomectomía y la EAU son dos procedimientos terapéuticos diferentes y de difícil comparación. Es un estudio susceptible de sesgo debido a las diferencias de selección y seguimiento. También hay que tener en cuenta que no son grupos homogéneos en cuanto a la edad media y deseo genésico.

La principal indicación de la miomectomía fue la sintomatología compresiva y de la EAU el sangrado anormal. Estos datos son similares a lo observado en otros estudios publicados (1).

La tasa de éxito a corto plazo en nuestro estudio, medida por la mejoría de los síntomas, fue del $84,1 \%$ para la miomectomía y del $92,3 \%$ para la EAU, similar a lo publicado en una revisión Cochrane, en donde se recogía una tasa de miomectomía efectiva del $93 \%$ y del $87,5 \%$ en la embolización (1). Así mismo, se han evaluado estos resultados a corto y largo plazo (a los 5-7 años) en el caso de la EAU datándose una mejoría clínica del 90 y $75 \%$ respectivamente (4).

Se registró una mayor tasa de complicaciones en el grupo de la miomectomía $(15,2 \%)$ respecto a la EAU (4,5\%). Aunque en este segundo grupo fue necesaria la reintervención en el 5,1\% (reembolización 1,3\% e histerectomía 3,8\%). Se ha publicado que la tasa general de complicaciones de la EAU 
es de un $10,5 \%$, y la tasa de complicaciones graves es de un 1,5\% (5). En una de las últimas revisiones realizadas, se recoge que los resultados a mediolargo plazo con ambas técnicas son similares, excepto una mayor frecuencia de reintervención en el grupo de la EAU (6). En nuestro estudio al igual que en otros, existe una diferencia estadísticamente significativa en la duración de la estancia hospitalaria a favor de la EAU (1).

Hubo 5 gestaciones tras la miomectomía (tres de las cuales llegaron a término) y ninguna en el grupo de la EAU. Sin embargo, hay que tener en cuenta que los grupos a estudio no son comparables en cuanto a la edad media, la paridad y el deseo genésico. La embolización de las arterias uterinas está asociada a mayores tasas de aborto, alteraciones placentarias, malposiciones fetales, restricciones de crecimiento fetal y prematuridad (3). La mayoría de los trabajos concluyen que la miomectomía es la mejor opción terapéutica en las mujeres con deseo genésico y miomas uterinos sintomáticos $(2,7,8)$. Aún así, en determinadas pacientes, para las que la única solución terapéutica es la histerectomía o una miomectomía de riesgo, podría plantearse la realización de una EAU como opción terapéutica.

Otro aspecto destacable es que en la mayoría de los estudios no hacen referencia a algunas variables importantes tales como el coste-beneficio y resultados comparativos con técnicas quirúrgicas menos invasivas.

A pesar de haberse registrado una mayor tasa de reintervención en las pacientes sometidas a EAU, este procedimiento debe tenerse en cuenta como un arma terapéutica en pacientes con alto riesgo quirúrgico y en aquellas en las que se quiera evitar una cirugía abdominal. Sería interesante realizar estudios que comparen otras opciones terapéuticas como la ablación endometrial, histerectomía total laparoscópica o la histerectomía vaginal.

\section{CONCLUSIÓN}

La adecuada indicación de cada técnica es fundamental para tener una baja tasa de complicacio- nes. Se deberá tener en cuenta la sintomatología, el tipo y número de miomas, la edad y el deseo genésico de cada paciente. La EAU debe considerarse como un arma terapéutica en pacientes de alto riesgo quirúrgico. La embolización realizada previamente a la miomectomía (preoperatoriamente o como combinación de ambas técnicas) puede ser una propuesta a considerar en casos seleccionados. Son necesarios más estudios que evalúen los efectos a largo plazo y el impacto en la fertilidad.

\section{REFERENCIAS}

1. Gupta JK, Sinha AS, Lumsden MA, Hickey M. Embolización de la arteria uterina para los fibromas uterinos sintomáticos (Revisión Cochrane traducida). En: La Biblioteca Cochrane Plus, 2008. Número 4. Oxford: Update Software Ltd. Disponible en: http://www. update-software.com. (Traducida de The Cochrane Library, 2008 Issue 3. Chichester, UK: John Wiley \& Sons, Ltd).

2. Mara M, Maskova J, Fucikova Z, Kuzel D, Belsan T, Sosna O. Midterm Clinical and First reproductive results of a randomized controlled trial comparing uterine fibroid embolization and myomectomy. Cardiovasc Intervent Radiol 2008;31:73-85.

3. Goldberg J, Pereira L, Berghella V, Diamond J, Daray E, Seinera $P$, Seracchioli $R$. Pregnancy outcomes after treatment for fibromyomata: uterine artery versus laparoscopy myomectomy. Am J Obstet Gynecol 2004; 191:18-21.

4. Kahn V, Fohlen A, Pelage JP. Role of embolization in the management of uterine fibroids .Gynecol Obstet Biol Reprod 2011;40:918-27.

5. Coleman P, Ayiku L. Systematic review of the efficacy and safety of uterine artery embolization in the treatment of fibroids. Sheffield: Review Body for Interventional Procedures; 2004.Commissioned by the National Institute for Clinical Excellence.

6. van der Kooij SM, Bipat S, Hehenkamp WJ, Ankum WM, Reekers JA. Uterine artery embolization versus surgery in the treatment of symptomatic fibroids: a systematic review and metaanalysis. Am J Obstet Gynecol 2011; 205:1-18.

7. Tulandi T, Salamah K. Fertility and uterine artery embolization. Obstet Gynecol 2010;115:857-60.

8. Sabrià E, Ponce J, Giné L, Plá MJ, Dib Al Tamr Al Barazi. Miomas, fertilidad y gestación. Ginecología Obstetricia Clínica 2009;10:21-6. 